\title{
Search for dark matter with jets and missing transverse energy at $13 \mathrm{TeV}$
}

\section{David Vannerom*广}

Interuniversity Institute for High Energies, IIHE (ULB-VUB)

Université Libre de Bruxelles, ULB - Vrije Universiteit Brussel, VUB

E-mail: david.vanneromecern.ch

\begin{abstract}
A search for new physics is performed using events having large missing transverse momentum and one or more jets with high transverse momenta in a data sample of proton-proton interactions at the centre-of-mass energy of $13 \mathrm{TeV}$. The data correspond to an integrated luminosity of 2.3 $\mathrm{fb}^{-1}$ collected in 2015 by the CMS detector, during the Run2 of the LHC. Results are presented in terms of limits on dark matter production based on simplified models.
\end{abstract}

Fourth Annual Large Hadron Collider Physics

13-18 June 2016

Lund, Sweden

*Speaker.

† On behalf of the CMS collaboration 


\section{Introduction}

Many theories predict the existence of Dark Matter (DM) particles interacting with Standard Model (SM) particles via bosonic mediators. This means DM could be produced at the LHC in proton-proton collisions. As DM particles would escape the detector without interacting, additional objects need to be produced along with the DM, this can be realized by initial state radiation (ISR, see figure 1). ISR of a gluon or a vector boson would result in spectacular events where at least one jet balances large missing transverse energy $\left(E_{T}^{\text {miss }}\right)$. In this poster proceedings, we will focus on gluon emission: these events are known as monojet events. Reference [1] presents the results for both scalar and vector mediators but we will focus on the vector mediator case only. Reference [2] is the standard reference for the CMS detector.
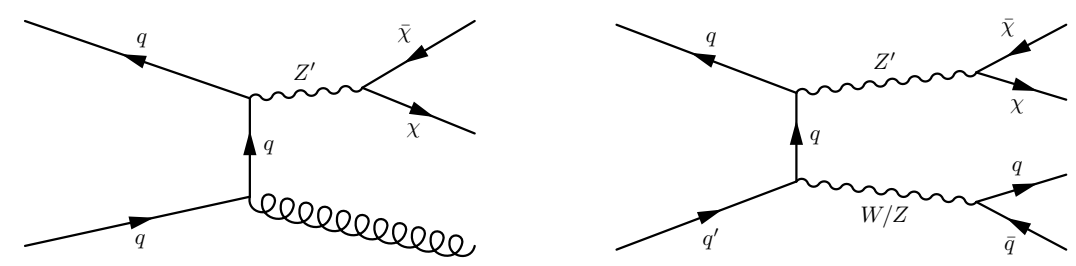

Figure 1: Feynman diagram for the production of a pair of DM particles recoiling against a gluon (left) or a hadronically decaying weak boson (right).

\section{Event selection}

The first selection step comes at the trigger level (online selection). The triggers used in this analysis require the following:

- $E_{T}^{\text {miss }, \mu}>90 \mathrm{GeV}$, where $E_{T}^{\text {miss }, \mu}$ is defined as the $E_{T}^{\text {miss }}$ with muons removed from the calculation. The events containing muons are therefore kept. They will be needed to estimate the two main backgrounds (see section Background Estimation).

- $H_{T}^{\text {miss }}>90 \mathrm{GeV}$, where $H_{T}^{\text {miss }}$ is defined as the $E_{T}^{\text {miss }}$ with only jets entering the calculation.

Then a further offline selection is applied. The main criteria are summarized in table 1.

\begin{tabular}{c|c|c} 
Variable & Cut & Purpose \\
\hline$E_{T}^{\text {miss }}$ & $>200 \mathrm{GeV}$ & Trigger efficiency $>98 \%$ above this threshold \\
$\mathrm{N}_{\text {jets }}$ & $\geq 1$ & Signal signature \\
Leading jet $p_{T}$ & $>100 \mathrm{GeV}$ & High energy events selection \\
$\Delta \phi\left(E_{T}^{\text {miss }}\right.$, jets $)$ & $>0.5$ & QCD background rejection \\
$N_{\text {lepton }}, N_{\text {photon }}$ and $N_{\text {b-jet }}$ & $=0$ & Electroweak and top quark background rejection
\end{tabular}

Table 1: Main selection criteria in the signal region. 
What is referred to as jets in the above selection are objects reconstructed using the anti- $k_{T}$ algorithm with a distance parameter of 0.4 (AK4 jets). Only jets with $p_{T}>30 \mathrm{GeV}$ are considered.

To control the $E_{T}^{\text {miss }}$ distribution is essential to this analysis. All suspicious or non-healthy events must therefore be cleaned out. In addition to the standard filters, we apply cuts on the energy fractions of the leading jet (see figure 2), i.e. we ask for a charged hadronic fraction $>$ 0.1 and a neutral hadronic fraction $<0.8$. The purpose of those two additional cuts is to remove beam halo events that have not been filtered out by the dedicated filtered. Beam halo events consist of muons created in the beam pipe, ahead of the collision point, flying through the detector along the beam axis. Those muons deposit energy in the calorimeters but not in the tracker system: this may result in a neutral hadron-like signal. This effect can be seen on figure 2 where we observe an excess of events in data at high neutral hadron fraction (or low charged hadron fraction).
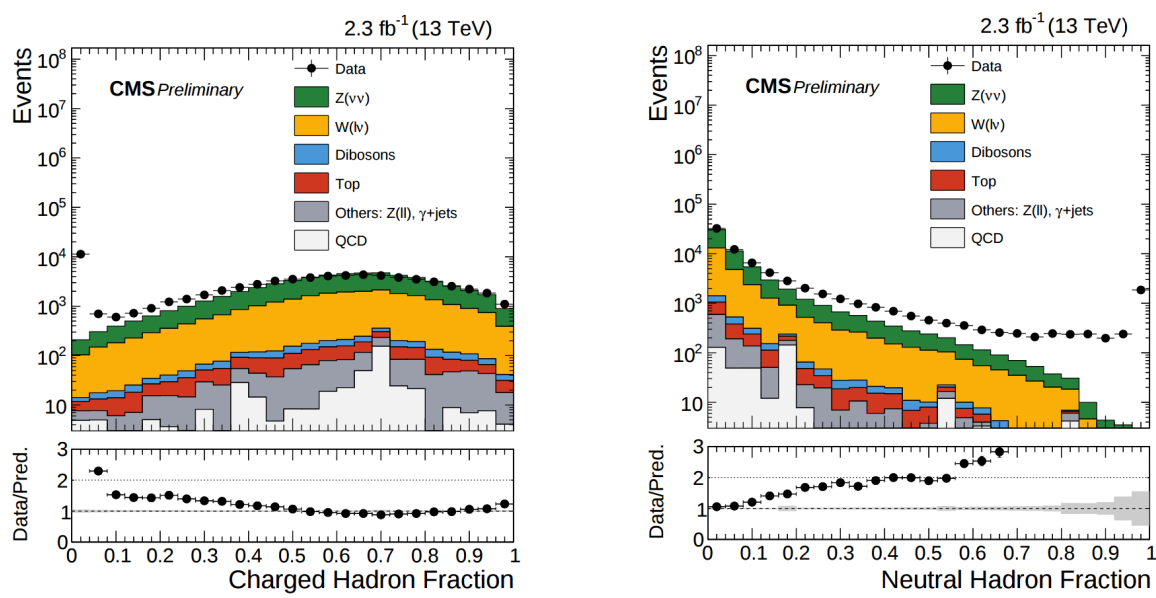

Figure 2: Charged (left) and neutral (right) hadronic fractions of the leading jet in data and in simulations.

\section{Event display}

Figure 3 shows two different views of the highest $E_{T}^{\text {miss }}$ monojet event passing the selection. This event has a $E_{T}^{\text {miss }}$ of $1467 \mathrm{GeV}$ and a a jet energy of $1466 \mathrm{GeV}$.

\section{Background estimation}

The DM signal is extracted from the $E_{T}^{\text {miss }}$ distribution using a shape-based method. The main issue of this analysis is the SM background estimation. The two main backgrounds are $Z(v v)$ and $W(l v)$ and this part only treats those two. The less contributing backgrounds are QCD, top quark and diboson. The contribution of the $Z(v v)$ background to the expected $E_{T}^{\text {miss }}$ distribution in the signal region is estimated using 5 control regions in data: $Z(\mu \mu)$ and $Z(e e)$ (dilepton), $W(\mu v)$ and $W(e v)$ (single-lepton) and $\gamma+$ jets. The use of single-lepton and $\gamma+$ jets regions allows to balance for the low $(\sim 1 / 10)$ statistics in the dilepton regions due to low branching ratios of $Z(l l)$ w.r.t. $Z(v v) . W(l v)$ background is estimated using only single-lepton regions. In the control regions, 

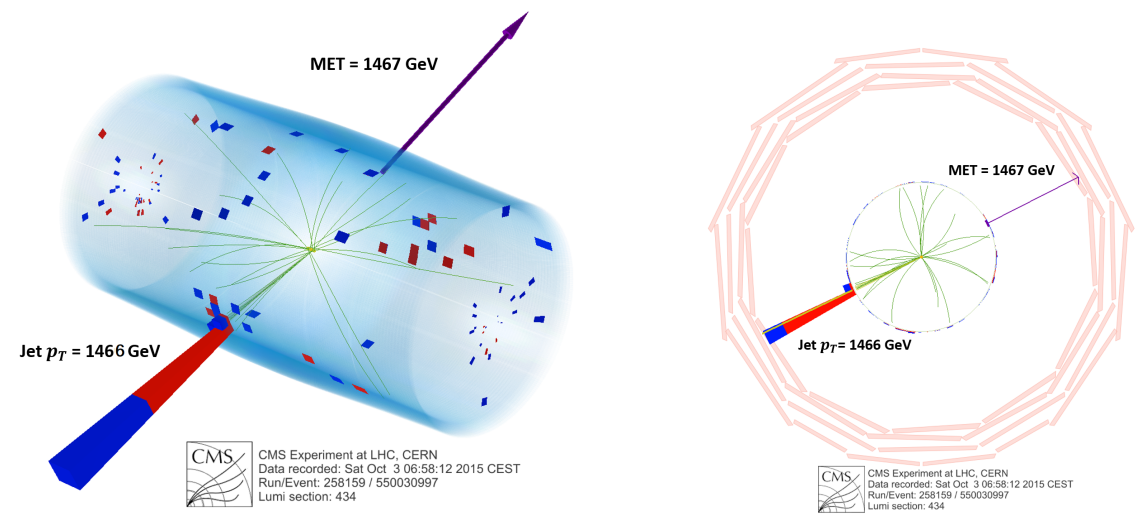

Figure 3: 3D (left) and transverse (right) view of the highest $E_{T}^{m i s s}$ event passing the selection.

the variable of interest is the hadronic recoil $\mathscr{U}$ which is defined as the $E_{T}^{\text {miss }}$ computed without the leptons (or the photon). The main selection criteria in the control regions are:

- Minimum $p_{T}$ requirements on photon and single leptons to ensure good trigger efficiency

- Same requirements on the recoil $\mathscr{U}$ as the ones applied on $E_{T}^{m i s s}$ in the signal region

Moreover, $M_{l l}^{i n v}$ must fall in $[60,120] \mathrm{GeV}$ for the dilepton regions. Figure 4 shows the recoil distributions for two control regions. Then, transfer factors are used to relate, bin by bin, the number of events in the $\mathscr{U}$ distribution in the control regions to that in the $E_{T}^{\text {miss }}$ distribution in the signal region. For example $R_{i}^{Z}=N_{i, M C}^{Z \rightarrow \mu \mu} / N_{i, M C}^{Z \rightarrow \nu v}$, in the case of $Z \rightarrow \mu \mu$ to $Z \rightarrow v v$. They account for differences in branching ratios, lepton acceptances and selection efficiencies. Finally, the bins of the recoil $\left(E_{T}^{\text {miss }}\right)$ distribution in the control (signal) region are re-weighted using a maximum likelihood fit including all five control regions and the signal region.
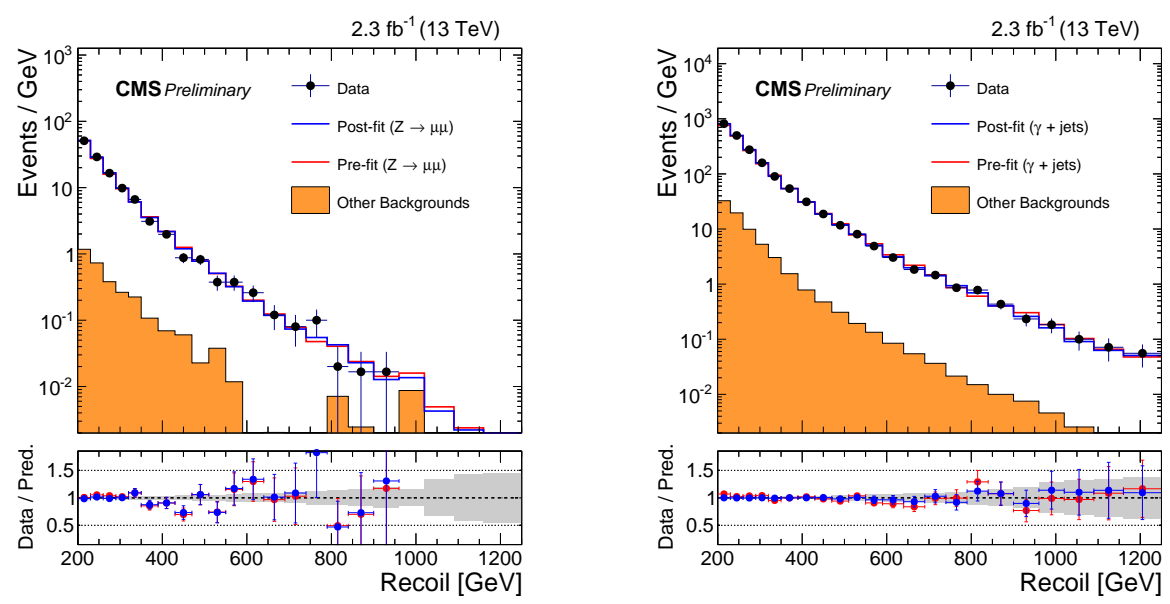

Figure 4: Recoil distribution in the dimuon (left) and the $\gamma+$ jets (right) control regions for the monojet category. We observe a better agreement between data and simulations in the $\gamma+$ jets region, which will help constraint the background estimation. 


\section{Results}

The data are consistent with the SM expectation as can be seen from the $E_{T}^{\text {miss }}$ distribution on figure 5. Exclusion limits are then determined in the context of simplified models: DM is modelled as fermionic particles interacting with SM particles via a spin-1 mediator. Figure 6 (left) presents the results in a two dimensional plane: the mass of the DM particles $\left(\mathrm{m}_{\mathrm{DM}}\right)$ and the mediator mass ( $\mathrm{m}_{\mathrm{MED}}$ ). Mediator masses up to $1.3 \mathrm{TeV}$ are excluded for vector models. Upper limits are set on the spin-independent DM-nucleon scattering cross-section $\left(\sigma_{\mathrm{SI}}\right)$ and compared to direct and indirect detection experiment results (see figure 6 (right)).

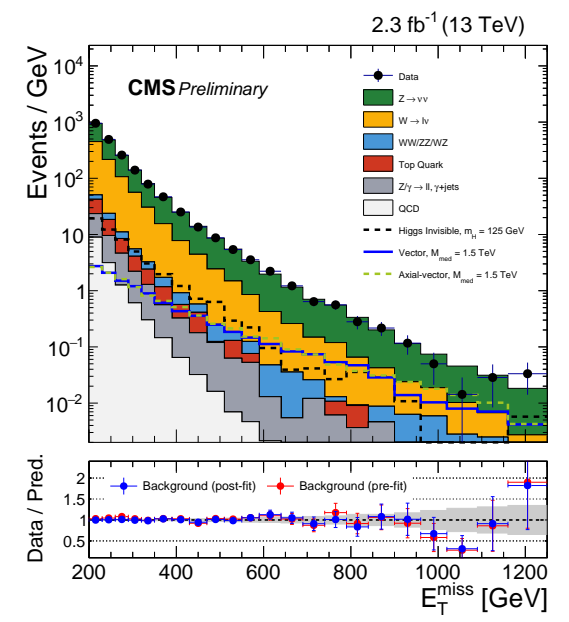

Figure 5: Post-fit distribution of the $E_{T}^{m i s s}$ for SM backgrounds, expected signal and data.
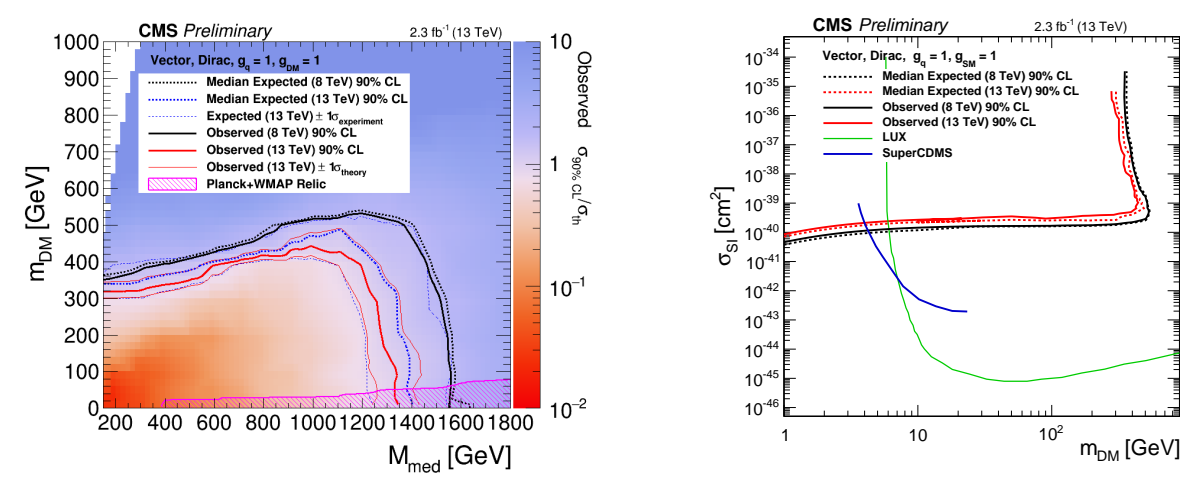

Figure 6: Exclusion limits at $90 \% \mathrm{CL}$ on $\mu=\sigma / \sigma_{t h}$ in the $\mathrm{m}_{\mathrm{DM}}-\mathrm{m}_{\mathrm{MED}}$ plane (left) and exclusion limits at $90 \% \mathrm{CL}$ on $\sigma_{\mathrm{SI}}$ in the $\mathrm{m}_{\mathrm{DM}}-\sigma_{\mathrm{SI}}$ plane (right) assuming vector mediator.

\section{References}

[1] The CMS collaboration, Search for dark matter production in association with jets or hadronically decaying $W$ or $Z$ boson at $\sqrt{s}=13 \mathrm{TeV}, 2016$, CMS Physics Analysis Summary (CMS PAS EXO-16-013).

[2] The CMS collaboration, The CMS experiment at the CERN LHC, 2008, JINST 3:S08004 